\title{
FTIR, Dissolution and Anti-viral Activity of Nevirapine Co-crystals \\ Samsodien $\mathrm{H}^{*}$, Bapoo M, Doms TI, Harneker Z, Louw AS, Scheepers IC, Sonday AB and Geldenhuys B
}

School of Pharmacy, University of the Western Cape, Bellville, South Africa

${ }^{*}$ Corresponding author: Dr. Halima Samsodien, Head of Pharmaceutics, School of Pharmacy, University of the Western Cape, Bellville, South Africa, Tel: 021-959-2179; Fax: 021-959-3407; E-mail: hsamsodien@uwc.ac.za

Received date: Jun 09, 2017; Accepted date: Sep 17, 2017; Published date: Sep 26, 2017

Copyright: @ 2017 Samsodien H, et al. This is an open-access article distributed under the terms of the Creative Commons Attribution License, which permits unrestricted use, distribution, and reproduction in any medium, provided the original author and source are credited.

\begin{abstract}
The study uses Fourier Transform Infrared (FTIR) spectroscopy to identify five Nevirapine (NV) co-crystals, determines the dissolution profile of the co-crystals and the antiviral activity comparative to pure NV.

Hot stage microscopy measured the purity and integrity of each co-crystal. FTIR analysis was used to identify the co-crystals to make recommendations regarding the future use of the technique to identify the NV co-crystals. Dissolution studies of the NV co-crystals prepared with maleic acid, salicylic acid and glutaric acid (NVMLE, NVSLI and NVGLT, respectively) were completed using the rotating basket method. Assays were conducted using High Performance Liquid Chromatography and compared to pure NV and the five NV: co-former mixtures. The antiviral

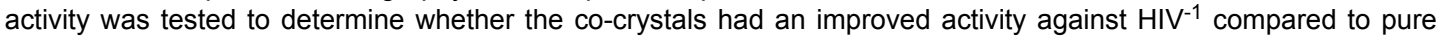
NV.

All co-crystals, except NVTTA (a NV co-crystal prepared with rac-tartaric acid), were pure and maintained their integrity for approximately one year. NVGLT, NVMLE and NVTTA, 1:1 molar ratio co-crystals were identified by FTIR. The $\mathrm{C}=\mathrm{O}$ stretching frequency of the carboxylic acid groups of NV and GLT were observed at $1638.15 \mathrm{~cm}^{-1}$ and $1719.23 \mathrm{~cm}^{-1}$ in the NVGLT co-crystal which corresponded with spectra of NVMLE and NVTTA. In NVMLE the $\mathrm{C}=\mathrm{O}$ stretching frequency of the $\mathrm{C}=\mathrm{O}$ of $\mathrm{NV}$ and MLE were observed at $1640.58 \mathrm{~cm}^{-1}$ and $1694.10 \mathrm{~cm}^{-1}$ and in NVTTA it was at $1637.25 \mathrm{~cm}^{-1}$ and $1708.50 \mathrm{~cm}^{-1}$, suggesting the presence of both parent molecules in the new phase for NVGLT, NVMLE and NVTTA.

Dissolution studies suggested that NVGLT was the only co-crystal that yielded better results than both NV and its physical mixture. The antiviral activity of the NVSC (an NV co-crystal prepared with saccharin) and NVSLI cocrystals in DMSO was significantly different to pure NV, demonstrating an improvement in anti-viral activity.

Keywords: Co-crystals; Nevirapine; Dissolution rate; Scale-up; Antiviral activity; FTIR; Solubility

\section{Introduction}

Nevirapine (NV) is a non-nucleoside reverse transcriptase inhibitor used in combination with other antiretroviral drugs for the treatment of Human Immunodeficiency Virus (HIV) infections. NV directly inhibits reverse transcriptase activity therefore suppressing DNA replication of the HIV virus and is known to prevent HIV transmission from mother to infant. A single dose of NV administered to the mother at the onset of labour and to the baby within 72 hours of delivery nearly halved the rate of HIV transmission. Since NV is given only once to the mother and baby it is relatively cheap and easy to administer [1-3].

$\mathrm{NV}$ is practically insoluble in water with an aqueous solubility of 0.1 $\mathrm{mg} / \mathrm{ml}^{-1}$ ( $\mathrm{pH} 7$, Temp. $37^{\circ} \mathrm{C}$ ). According to the Biopharmaceutical Classification Index, NV is a Class II drug i.e. it has a high permeability and a low solubility [1]. The low rate of dissolution of NV is assumed to be the rate-limiting step for absorption of the drug [4].

Co-crystals, a crystalline structure, containing two or more different components in a definite stoichiometric ratio was investigated to enhance the solubility, bioavailability and the dissolution rate of NV. Co-crystals are formed between a molecular or ionic active pharmaceutical ingredient (API) and a co-crystal former, where each component is a solid at ambient temperature and produces a solid product at ambient temperature as well [5]. Co-formers were selected according to hydrogen bonding rules to facilitate non-covalent bonding between molecules. NV co-crystals were formed with Generally-Regarded-As-Safe (GRAS) compounds, namely Saccharin (SC), Tac-Tartaric Acid (TTA), Maleic Acid (MLE) and Salicylic Acid (SLI). Glutaric Acid (GLT) was also used as a co-former to form NV co-crystals. NVSC and NVSLI formed co-crystals with a 2:1 ratio of $\mathrm{NV}$ to the relevant co-former. NVTTA, NVMLE and NVGLT formed co-crystals with a 1:1 ratio of NV to the relevant co-former [1].

In a similar study, the formulation of nicotinamide-based cocrystals of fenofibrate by different methods in 1:1 molar ratio were used to formulate molecular complexes by kneading, solution crystallization, antisolvent addition and solvent drop grinding. The prepared molecular complexes were characterized by powder X-ray diffractometry, differential scanning calorimetry, Fourier transform infrared spectroscopy (FTIR), nuclear magnetic resonance spectroscopy and in vitro dissolution analysis. The analytical techniques have all been widely employed to distinguish between different crystal forms such as polymorphs, clathrates, hydrates and co-crystals. FTIR has also been used to monitor co-crystal formation and single synthon detection. 
This study expands on the previous study by determining the dissolution of the three co-crystals not previously tested, namely NVMLE, NVSLI and NVGLT. FTIR spectroscopy was examined as a technique to identify the co-crystals. Finally, the five co-crystals were tested for their antiviral activity by the National Institute of Communicable Diseases (NICD).

\section{Materials and Methods}

\section{Hot stage microscopy}

All co-crystals, pure NV and co-formers were donated by the original research group responsible for their preparation Caira et al. [1]. The integrity and purity of all co-crystals were verified using hot stage microscopy (HSM) prior to experimentation.

A Linkam TH MS600 Temperature control stage connected to a T95 Linkpad System Controller was used to heat crystals at a controlled rate of $20^{\circ} \mathrm{C}$ per minute commencing at room temperature until degradation temperature of the product had been reached. Visual characterisation was captured by an Olympus UC30 colour video camera fitted to an Olympus SZX7 stereoscopic microscope. The recorded images were analysed by Stream Essentials ${ }^{\circledR}$ software.

\section{Fourier transform infrared spectroscopy}

Fourier Transform Infrared (FTIR) spectroscopy was conducted to confirm the identity of the five co-crystals and to determine whether FTIR could be used in future co-crystal studies to identify co-crystal forms.

IR spectra were collected for all five co-crystals, NV and each of the co-formers. Each sample was manually ground and a sufficient amount was placed on a PerkinElmer Spectrum 400 FTIR Spectrometer. The spectral range was between $400-4000 \mathrm{~cm}^{-1}$ and spectral resolution was $0.4-65 \mathrm{~cm}^{-1}$. Wavelength repeatability was $\pm 0.02 \mathrm{~cm}^{-1}$ at $1,600 \mathrm{~cm}^{-1}$ with wavelength accuracy being $\pm 0.1 \mathrm{~cm}^{-1}$ at $1,600 \mathrm{~cm}^{-1}$. All spectra were recorded in triplicate to ensure reproducibility of data. No preparation of sample was required due to the simplified analysis of powders and difficult solid materials using the PerkinElmer Spectrum 400 FTIR. Solid samples were placed directly onto the sample orifice and held in position with a strong steel handle. Sample sizes were between 5 to $10 \mathrm{mg}$ with no destruction of the sample. The spectra were produced and analysed using Spectrum software version 6.3.5.

\section{Dissolution testing}

Dissolution: Dissolution studies were conducted for NV alone, the co-crystals NVSLI, NVGLT and NVMLE, as well as separate mixes of NV with SLI, GLT and MLE (NV:SLI, NV:GLT and NV:MLE) in a 1:1 ratio. To ensure reproducibility these studies were conducted using the rotating basket method previously used by Caira et al. [1]. The powder mixtures and co-crystals were ground for 5 minutes before encapsulation to ensure a particle size range of $65-200 \mu \mathrm{m}$. Gelatin capsules were used to encapsulate $10 \mathrm{mg}$ of $\mathrm{NV}, 10 \mathrm{mg}$ of each of the 5 co-crystals and $10 \mathrm{mg}$ of each mixture at a 1:1 molar ratio.

Tests were carried out in a Distek evolution 6100 dissolution system with the temperature maintained at $37^{\circ} \mathrm{C}$. Flasks $(900 \mathrm{ml})$ filled with Reverse Osmotic water were used and a stirring speed of $100 \mathrm{rpm}$ was applied. The capsules were placed in stainless steel baskets to prevent floating. A 3 hour run was conducted with $5 \mathrm{ml}$ samples being filtered for analysis at 15 minute intervals. The assays were performed by HPLC.

\section{High performance liquid chromatography}

A standard curve of NV was generated as follows: $10 \mathrm{ml}$ of $3 \mathrm{M}$ HCL in 1 litre of distilled water was dissolved. To create a stock solution, $55.5 \mathrm{mg}$ of $\mathrm{NV}$ was dissolved in $100 \mathrm{ml}$ of this solution in a $100 \mathrm{ml}$ volumetric flask. $4 \mathrm{ml}$ of the stock solution was diluted to 200 $\mathrm{ml}$ with MilliQ water. The standard was performed in duplicate to ensure accuracy. A linearity test was performed using a serial dilution of the stock solution. A regression line with an $\mathrm{r}^{2}=0.9969$ was obtained. The HPLC assay for NV was achieved using an all-in-one system, the Shimadzu LC 2010 AHT system with Class VP software. The HPLC assays for the co-crystals and the mixes were performed using an Agilent 1200 system with Waters Empower software. The system consisted of an Auto injector with heater (G1316A), a Diode Array detector VL (G1329A) and a Quaternary pump (G1315D) as the solvent delivery module. The column used was a Phenomenex Luna $5 \mathrm{u}$ $\mathrm{C} 18,100 \mathrm{~A}$ and the flow rate was set to $1 \mathrm{ml}$ per minute with a column temperature maintained at $35^{\circ} \mathrm{C}$ [2]. The injection volume was set at 10 $\mu \mathrm{L}$. The mobile phase was prepared by dissolving $14.38 \mathrm{~g}$ of ammonium phosphate buffer with 4 litres of MilliQ water. This was stirred with a magnetic stirrer until the ammonium phosphate buffer had dissolved. The buffer was placed into a 5 litre volumetric flask and the $\mathrm{pH}$ was adjusted to 5 using $1 \mathrm{M} \mathrm{NaOH}$. The solution was then made up to 5 litres using MilliQ water. The mobile phase was filtered through a $0.45 \mu \mathrm{m}$ filter with compressed air.

\section{Antiviral testing}

Antiviral testing on the five co-crystals were carried out at the National Institute of Communicable Diseases (NICD).

Cytotoxicity Screen: Toxicity screening was conducted to determine if the co-crystals were cytotoxic towards the NICD's specific 293T cells. Toxicity can be reflected by cell viability. This is measured by the bioreduction of a tetrazolium compound, Thiazolyl Blue Tetrazolium Bromide (MTT) (Sigma), to a coloured formazan product in the culture medium. Dead cells do not cause this colour change. The formazan product is spectrophotometrically quantified and the degree of toxicity is related to the MTT-to-formazan conversion by the cells.

Stock solutions of each co-crystal and pure NV were prepared using dimethyl sulfoxide (DMSO) to contain an effective NV concentration of $10 \mathrm{mM}$. The stock solution was diluted to working concentrations in complete Dulbecco's Modified Eagle's medium (DMEM) with Fetal Bovine Serum (FBS), 4-(2-hydroxyethyl) ${ }^{-1}$-piperazineethanesulfonic acid (HEPES) and antibiotics. A dimethyl sulfoxide (DMSO) control solution was prepared which was run in parallel. The working solution was titrated into complete DMEM medium by serial dilution in a 96well culture plate, providing a range of concentrations for toxicity testing. A control cell was included. The culture plate was incubated for 2 days at $37^{\circ} \mathrm{C}$ under $5 \% \mathrm{CO}_{2}$ in a humidified atmosphere. MTT reagent was added and the plates were incubated for approximately 1 hr. A solution of sodium dodecyl sulphate (10\%) and dimethylformamide (50\%) was added to lyse the cells and dissolve the formazan crystals. The spectro-photometric absorbance of each well was read at $490 \mathrm{~nm}$, with $650 \mathrm{~nm}$ chosen as the reference wavelength. The percentage viability was then calculated. A viability of $0 \%$ indicates total cell death while a viability of $100 \%$ indicates full cell viability. 
Activity screen: The antiviral activity of the co-crystals to $\mathrm{HIV}^{-1}$ pseudovirus in relation to pure NV was tested. This assay investigated the inhibition of viral replication in a single cycle of infection. The pseudovirus contains $\mathrm{HIV}^{-1}$ sub-type $\mathrm{C}$ reverse transcriptase, integrase and protease as well as a RNA transcript of the firefly luciferase protein. During infection, reverse transcription of the RNA by the $\mathrm{HIV}^{-1}$ reverse transcriptase to complementary DNA occurs, which is then integrated into the host cell's DNA by $\mathrm{HIV}^{-1}$ integrase. The firefly luciferase gene is expressed to produce active firefly luciferase which can be assayed through a bioluminescence reaction. This reaction can be quantified. The signal is directly proportional to the number of infectious viruses in the inoculum. In the presence of an inhibitor, in this case NV, the number of firefly luciferase gene copies that are integrated into the genome will be reduced, thereby decreasing the amount of bioluminescence observed.

The non-toxic concentration of each co-crystal, as determined by the toxicity screen, was used to determine the activity. The co-crystal solution was diluted in complete DMEM medium to contain a final effective NV concentration of $18 \mu \mathrm{M}$, the highest non-toxic concentration. Eleven three-fold serial dilutions of each sample stock was prepared, starting at $18 \mu \mathrm{M}$, these dilutions were then titrated into a 96-well culture plate. Cells and virus were added and the plates were then incubated for 48 hours at $37^{\circ} \mathrm{C}$ under $5 \% \mathrm{CO} 2$ in a humidified atmosphere. The co-formers were diluted and prepared to concentrations which were similar to the co-crystal solutions. A virus control was included in the test, which contained only cells, virus and medium. A DMSO solvent control was also included. The antiviral activity of the co-crystals was screened using a standard $\mathrm{HIV}^{-1}$ subtype $\mathrm{C}$ isolate. Following incubation, Bright $\mathrm{Glo}^{\mathrm{ma}}$ Reagent was used to assay for the firefly luciferase in the wells. A luminometer was then used to quantify the bioluminescence emitted. The percentage of viral activity was then calculated. A viral activity of $0 \%$ would indicate complete viral inhibition, while that of $100 \%$ would indicate no inhibition. The inhibitory concentration-50 $\left(\mathrm{IC}_{50}\right)$ value indicates the concentration of co-crystal where $50 \%$ of the virus is inhibited. A dose-response curve was used to obtain these values. The activity screen was performed in duplicate.

The average and standard deviation of the $\mathrm{IC}_{50}$ of the two runs was calculated using Microsoft Excel. An unpaired two-tailed t-test with 95\% confidence intervals was performed using GraphPad Prism 4, in order to determine if a significant difference occurs between the viral activity of the co-crystals and pure NV.

\section{Results and Discussion}

\section{Hot stage microscopy}

In Figure $1 \mathrm{a}$ and $\mathrm{b}$, the first sign of melting for NVSC occurred at $223^{\circ} \mathrm{C}$ and complete melting was observed at $230^{\circ} \mathrm{C}$, this data corresponds to that achieved in the previous study. This result suggests that NVSC had maintained its integrity and purity and could be used in further experiments. According to M.R. Caira et al., NVTTA was expected to melt at $230^{\circ} \mathrm{C}$. The NVTTA tested began to spontaneously bubble at a temperature of $100^{\circ} \mathrm{C}$. A yellow discolouration appeared at a temperature of $140^{\circ} \mathrm{C}$. The spontaneous bubbling continued, with the co-crystal turning an orange-brown colour at $230^{\circ} \mathrm{C}$ (Figure $2 \mathrm{a}$ and b). The NVTTA had degraded; and could no longer be used for further experiments since the sample had lost its purity and integrity. A new batch of NVTTA was manufactured. All other co-crystals maintained their purity and integrity.
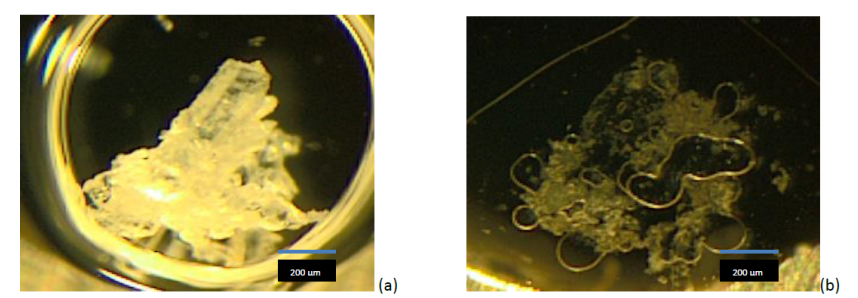

Figure 1: NVSC co-crystals at room temperature $\left(25^{\circ} \mathrm{C}\right)(\mathrm{a})$ and at $230^{\circ} \mathrm{C}(\mathrm{b})$.
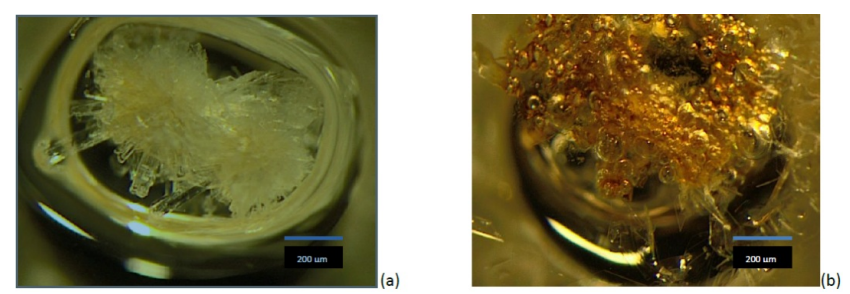

Figure 2: NVTTA co-crystals at $\left(25^{\circ} \mathrm{C}\right)$ and (b) $230^{\circ} \mathrm{C}$ showing brown discolouration.

\section{Fourier transform infrared spectroscopy}

The FTIR spectrum of each co-crystal was compared to NV and its relevant co-former. A wavelength shift of a peak or a change in peak intensities between the spectra would suggest that a co-crystal had formed. These peaks are a representation of the bonding points of the two molecules and are expected to occur at the $\mathrm{C}=\mathrm{O}, \mathrm{O}-\mathrm{H}$ and $\mathrm{N}-\mathrm{H}$ groups of the active pharmaceutical ingredient and co-former $\mathrm{C}=\mathrm{O}$ peaks occur between $1680^{-1} 750 \mathrm{~cm}^{-1}$ (Figure 3a-e). NV and all coformers peaks were identified in this region and compared in the cocrystal (Table 1). The FTIR spectrum of NV showed its $\mathrm{C}=\mathrm{O}$ stretch at $1643.64 \mathrm{~cm}^{-1}$. GLT, MLE and TTA, $\mathrm{C}=\mathrm{O}$ peaks appeared at 1686.05 $\mathrm{cm}^{-1}, 1704.09 \mathrm{~cm}^{-1}$ and $1723.98 \mathrm{~cm}^{-1}$, respectively (Table 1).

\begin{tabular}{|l|l|l|l|l|}
\hline & & NVGLT & NVMLE & NVTTA \\
\hline NV & $1643.64 \mathrm{~cm}^{-1}$ & $1638.15 \mathrm{~cm}^{-1}$ & $1640.58 \mathrm{~cm}^{-1}$ & $1637.25 \mathrm{~cm}^{-1}$ \\
\hline GLT & $1686.05 \mathrm{~cm}^{-1}$ & $1719.23 \mathrm{~cm}^{-1}$ & & \\
\hline MLE & $1704.09 \mathrm{~cm}^{-1}$ & & $1694.1 \mathrm{~cm}^{-1}$ & \\
\hline TTA & $1723.98 \mathrm{~cm}^{-1}$ & & & $1708.5 \mathrm{~cm}^{-1}$ \\
\hline
\end{tabular}

Table 1: Shifts observed in the $\mathrm{C}=\mathrm{O}$ peaks for NVGLT, NVMLE and NVTTA. 
Citation: Samsodien H, Bapoo M, Doms TI, Harneker Z, Louw AS, et al. (2017) FTIR, Dissolution and Anti-viral Activity of Nevirapine Cocrystals. Pharm Anal Acta 8: 561. doi:10.4172/2153-2435.1000561
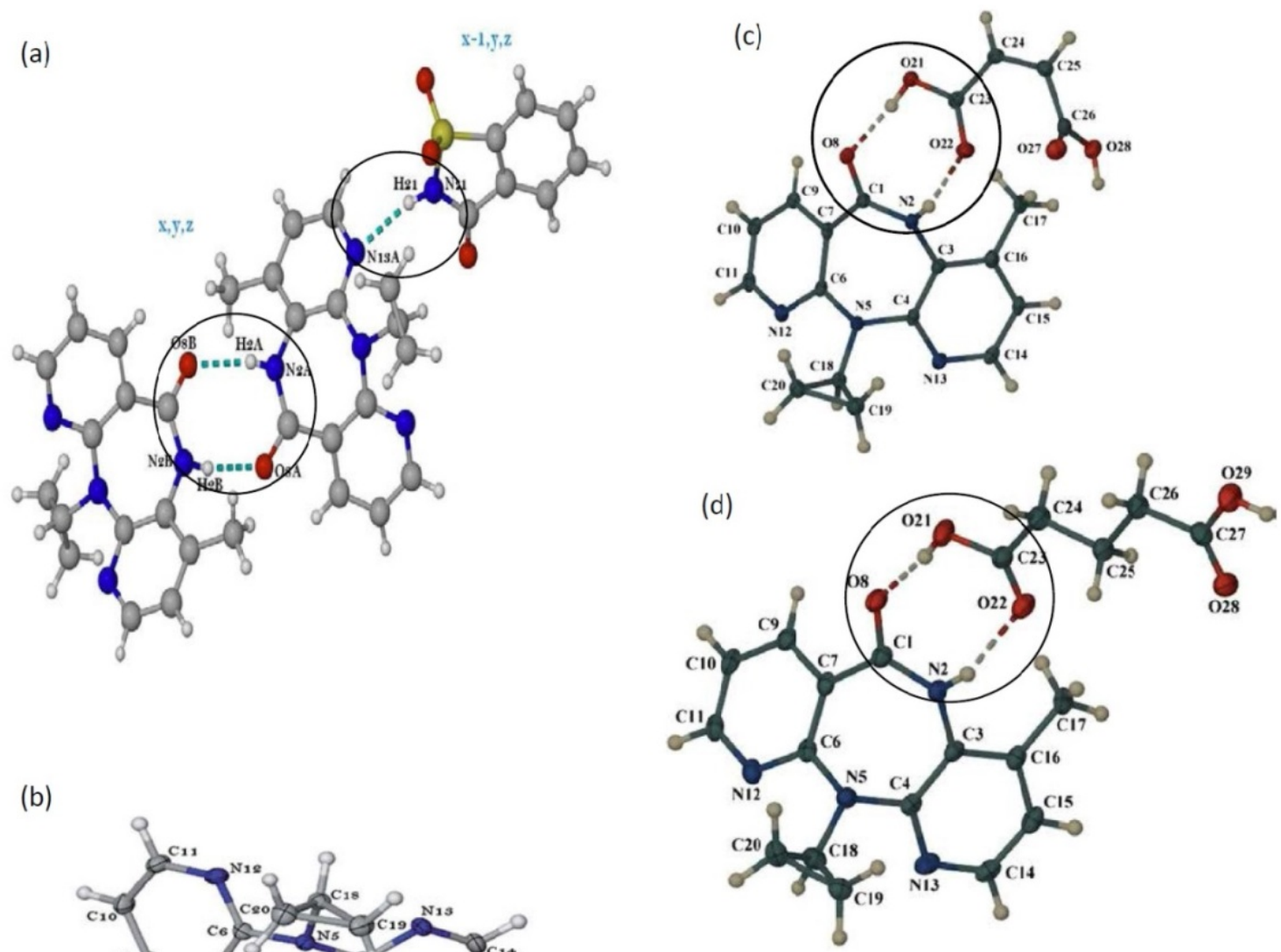

(b)
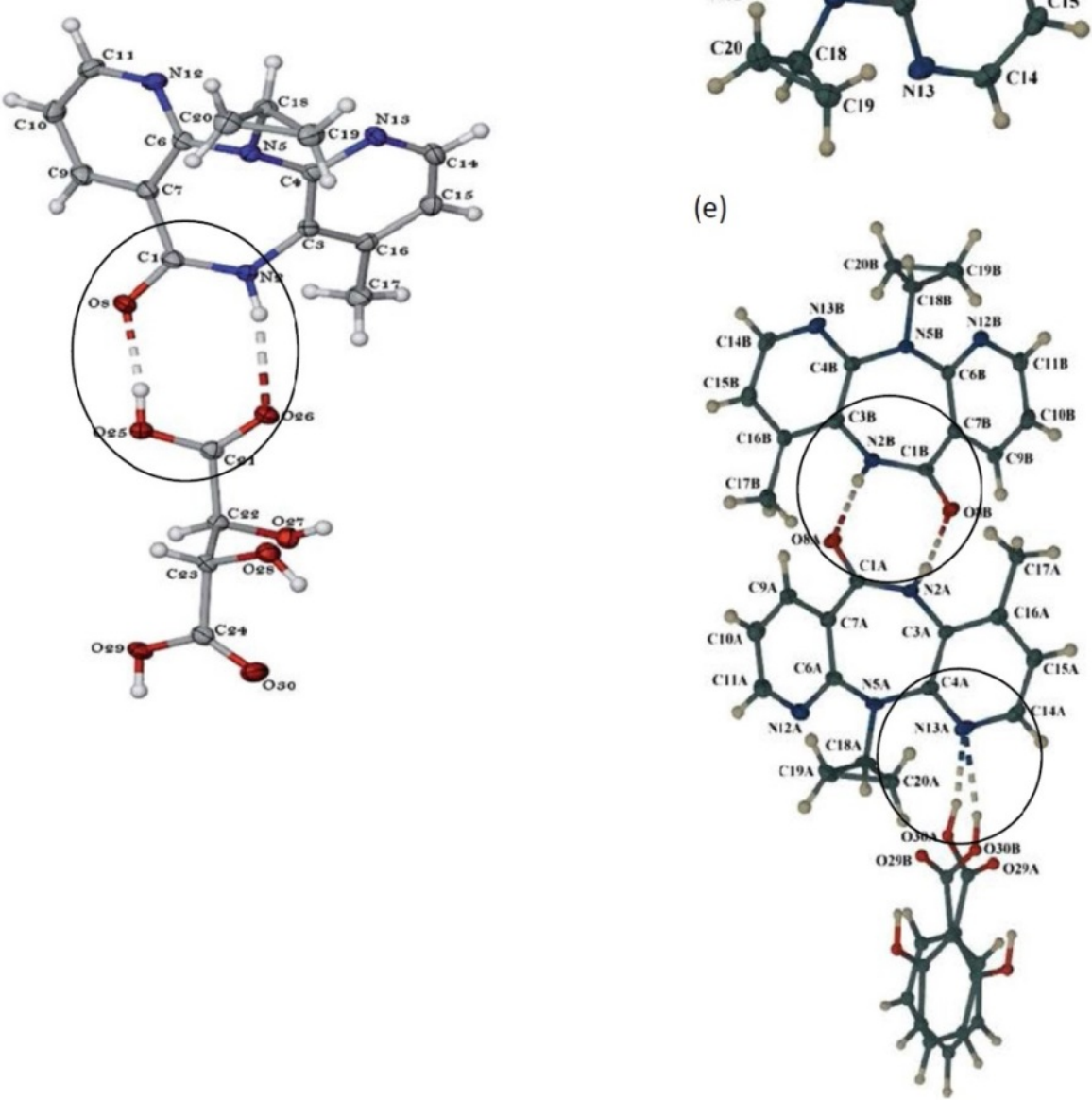

Figure 3: Co-crystals NVSC (a), NVTTA (b), NVMLE (c), NVGLT (d) and NVSLI (e) showing the bonds involved in the shift of the IR peaks. 
Citation: Samsodien H, Bapoo M, Doms TI, Harneker Z, Louw AS, et al. (2017) FTIR, Dissolution and Anti-viral Activity of Nevirapine Cocrystals. Pharm Anal Acta 8: 561. doi:10.4172/2153-2435.1000561

Page 5 of 10

The $\mathrm{C}=\mathrm{O}$ stretching frequency of the carboxylic acid groups of $\mathrm{NV}$ and GLT were observed at $1638.15 \mathrm{~cm}^{-1}$ and $1719.23 \mathrm{~cm}^{-1}$ in the NVGLT co-crystal (Figure 4a-c). This was also observed in the FTIR spectrum of NVMLE and NVTTA co-crystals. In NVMLE the $\mathrm{C}=\mathrm{O}$ stretching frequency of the carboxylic acid group of NV and MLE were observed at $1640.58 \mathrm{~cm}^{-1}$ and $1694.10 \mathrm{~cm}^{-1}$ (Figure $5 \mathrm{a}-\mathrm{c}$ ) and in NVTTA the $\mathrm{C}=\mathrm{O}$ stretching frequency of the carboxylic acid group of $\mathrm{NV}$ and TTA were observed at $1637.25 \mathrm{~cm}^{-1}$ and $1708.50 \mathrm{~cm}^{-1}$ (Figure $6 a-c)$. These results indicate the presence of both parent molecules in the new phase for NVGLT, NVMLE and NVTTA.

In a study by S. Basavoju et al. (2007)7 FTIR was used to identify cocrystals of indomethacin and SC by comparing the $\mathrm{C}=\mathrm{O}, \mathrm{O}-\mathrm{H}$ and $\mathrm{N}$ -
$\mathrm{H}$ peaks in the spectra of the co-crystal with those of the parent compounds. In this study the Spectrum software utilized (version 6.3.5) did not identify the $\mathrm{O}-\mathrm{H}$ or $\mathrm{N}-\mathrm{H}$ peaks as expected, however shifts and change in intensity of peaks were identified. These results in the FTIR spectra of SLI a C=O peak occurs at $1654.88 \mathrm{~cm}^{-1}$, however this peak is not represented in the spectra of the NVSLI co-crystal. This could be because the NVSLI co-crystal forms a 2:1 ratio, which could indicate that the $\mathrm{C}=\mathrm{O}$ bonds from the $2 \mathrm{NV}$ molecules are superimposed on the SLI's $\mathrm{C}=\mathrm{O}$ bond (Figure $7 \mathrm{a}-\mathrm{c}$ ). This phenomenon is also present in the NVSC co-crystal which also forms a 2:1 ratio. A shift in the $\mathrm{C}=\mathrm{O}$ bond from the co-former is not observed in the cocrystal (Figure 8a-c).

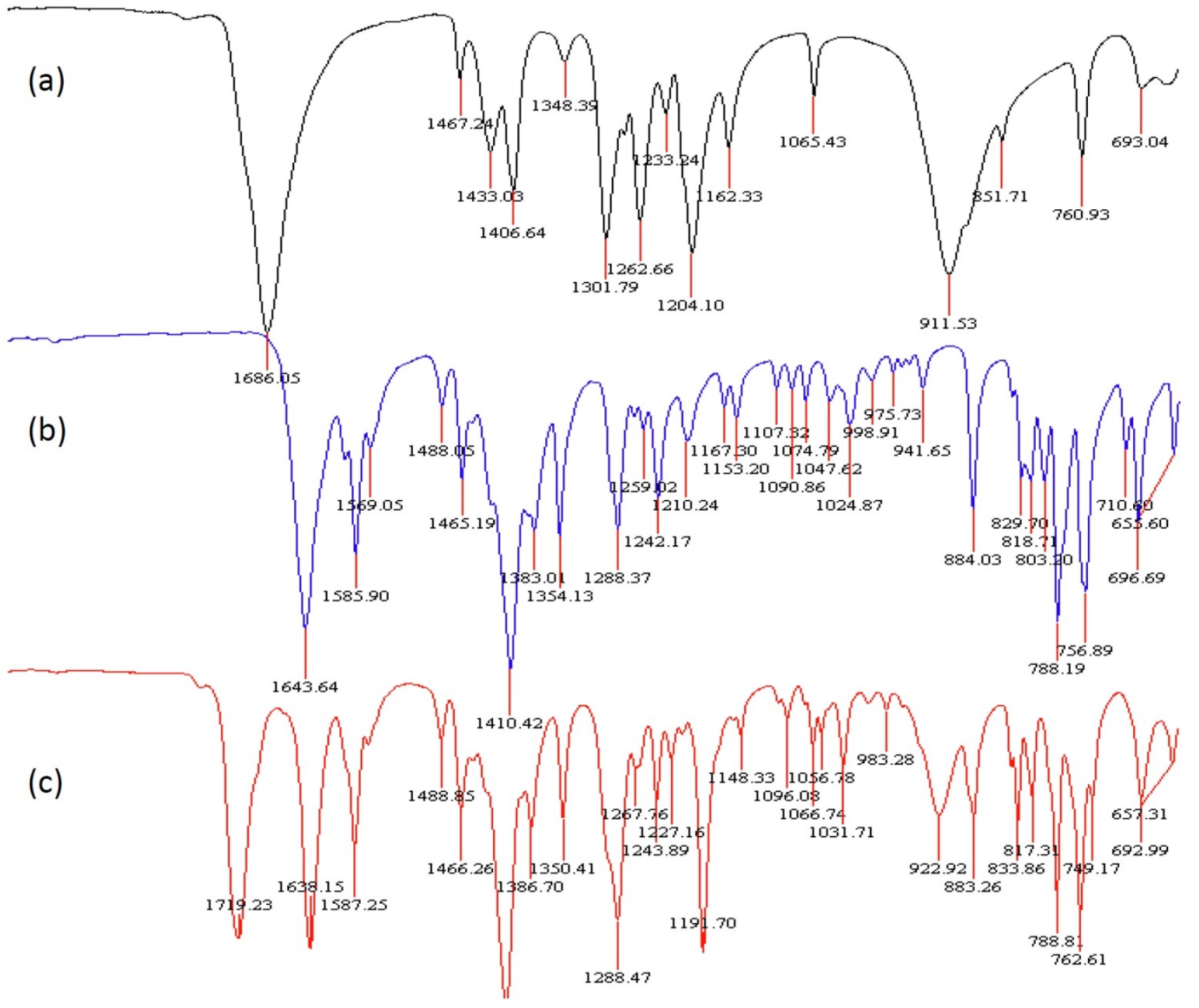

Figure 4: FTIR spectra of (a) GLT, (b) NV and (c) NVGLT co-crystals.

Following these results, we find that FTIR can be used for the identification of co-crystals creating a database of potential co-crystals with single X-ray diffraction analysis used as confirmation of the result. However, each API and relevant co-former will produce a different shift in the co-crystal and caution should be used when attempting to identify co-crystals when the co-crystal is known to form in a 2:1 ratio. The Spectrum software used should be able to 
Citation: Samsodien H, Bapoo M, Doms TI, Harneker Z, Louw AS, et al. (2017) FTIR, Dissolution and Anti-viral Activity of Nevirapine Cocrystals. Pharm Anal Acta 8: 561. doi:10.4172/2153-2435.1000561

Page 6 of 10

detect the bonds involved when the API and co-former bind to form the co-crystal (Figure 3).

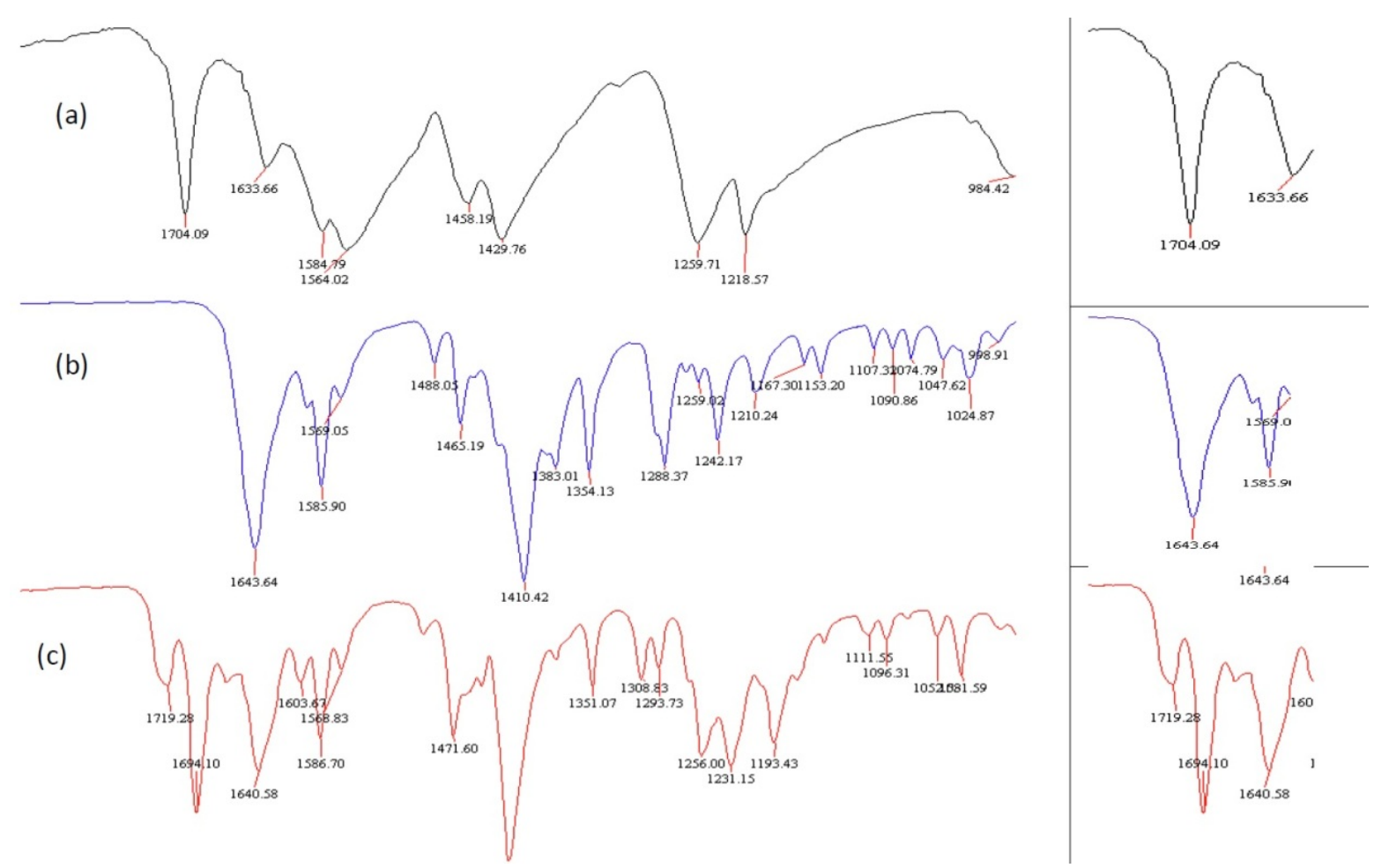

Figure 5: FTIR spectra of (a) MLE, (b) NV and (c) NVMLE co-crystals.

\section{Dissolution testing}

In vitro dissolution tests for co-crystals NVGLT, NVSLI and NVMLE were conducted to compare their dissolution to their respective NV-co-former mixtures and the untreated API. The dissolution profile (Figure 9) of NV improved for both the co-crystals and the physical mixtures. NVGLT was the only co-crystal that yielded better results than both NV and its physical mixture. However, NVGLT and its physical mixture still did not comply with the British Pharmacopoeia 2005 (BP) standards in that $75 \%$ of the substances did not go into solution within 45 minutes [6-8]. Only $30 \%$ and $26 \%$ went into solution after 45 minutes for NVGLT co-crystal and NV:GLT mixture, respectively. The NV:SLI and NV:MLE physical mixtures had better dissolution profiles compared to their co-crystals.

Caira et al. used the dissolution-time curves of NVSC and NVTTA to determine the solubility enhancement parameter of these cocrystals. It was estimated that the NVMLE co-crystal would produce the highest increase in aqueous solubility of NV, that of a five-fold increase. The experimental dissolution results disprove this as only $39 \%$ of NVMLE went into solution after 180 minutes, corresponding to a two-fold increase. In comparison, 54\% of NV:MLE went into solution after 180 minutes.
The increase in aqueous solubility of NV produced by NV: SLI was twice as much as the increase produced by NVSLI. NVSLI had a total dissolution of only $32 \%$ while NV: SLI had a total dissolution of $65 \%$. The NV: SLI physical mixture had the best dissolution profile, producing the highest percentage total dissolution and having the fastest rate of dissolution compared to all other samples.

As seen by Figure 9, NV alone had a total dissolution of only $16 \%$. This was not expected, since Caira et al. showed a dissolution of approximately $60 \%$. It is important to note that $100 \%$ pure NV was used, and the NV used in both studies was from the same batch. The discrepancy in the dissolution profiles of NV could be due to the fact that different analytical methods were used for the dissolution assay. The previous study used UV spectrophotometry at $234 \mathrm{~nm} 1$, while HPLC was used in this study. UV spectra show peaks at varying wavelengths but only one is chosen and thus does not have the same degree of specificity as HPLC. In comparison, HPLC produces results for a distinct retention time only, and is thus more precise and accurate. No validation was done to prove that the methods would produce similar results. 
Citation: Samsodien H, Bapoo M, Doms TI, Harneker Z, Louw AS, et al. (2017) FTIR, Dissolution and Anti-viral Activity of Nevirapine Cocrystals. Pharm Anal Acta 8: 561. doi:10.4172/2153-2435.1000561

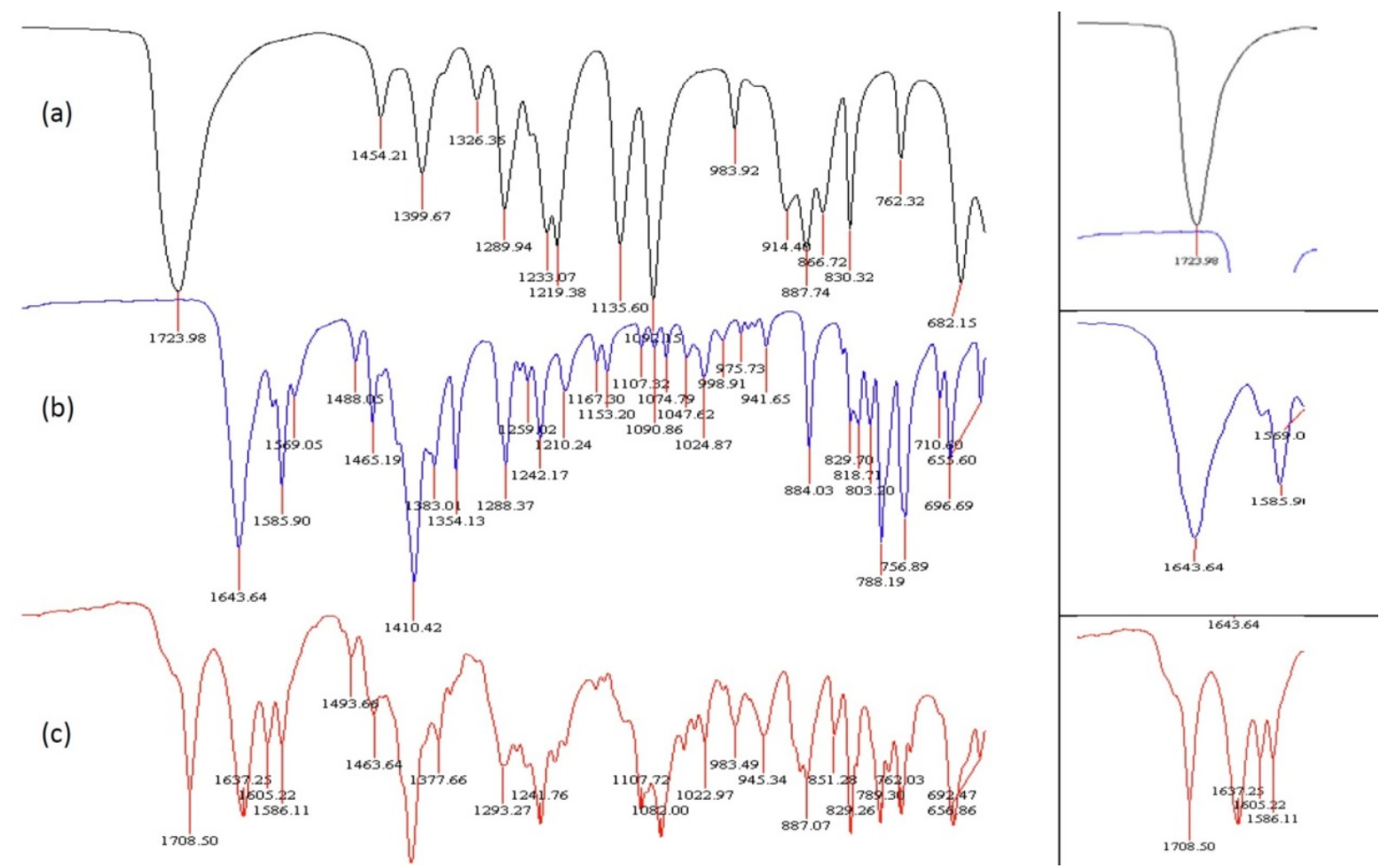

Figure 6: FTIR spectra of (a) TTA (b) NV and (c) NVTTA co-crystals.

(a)
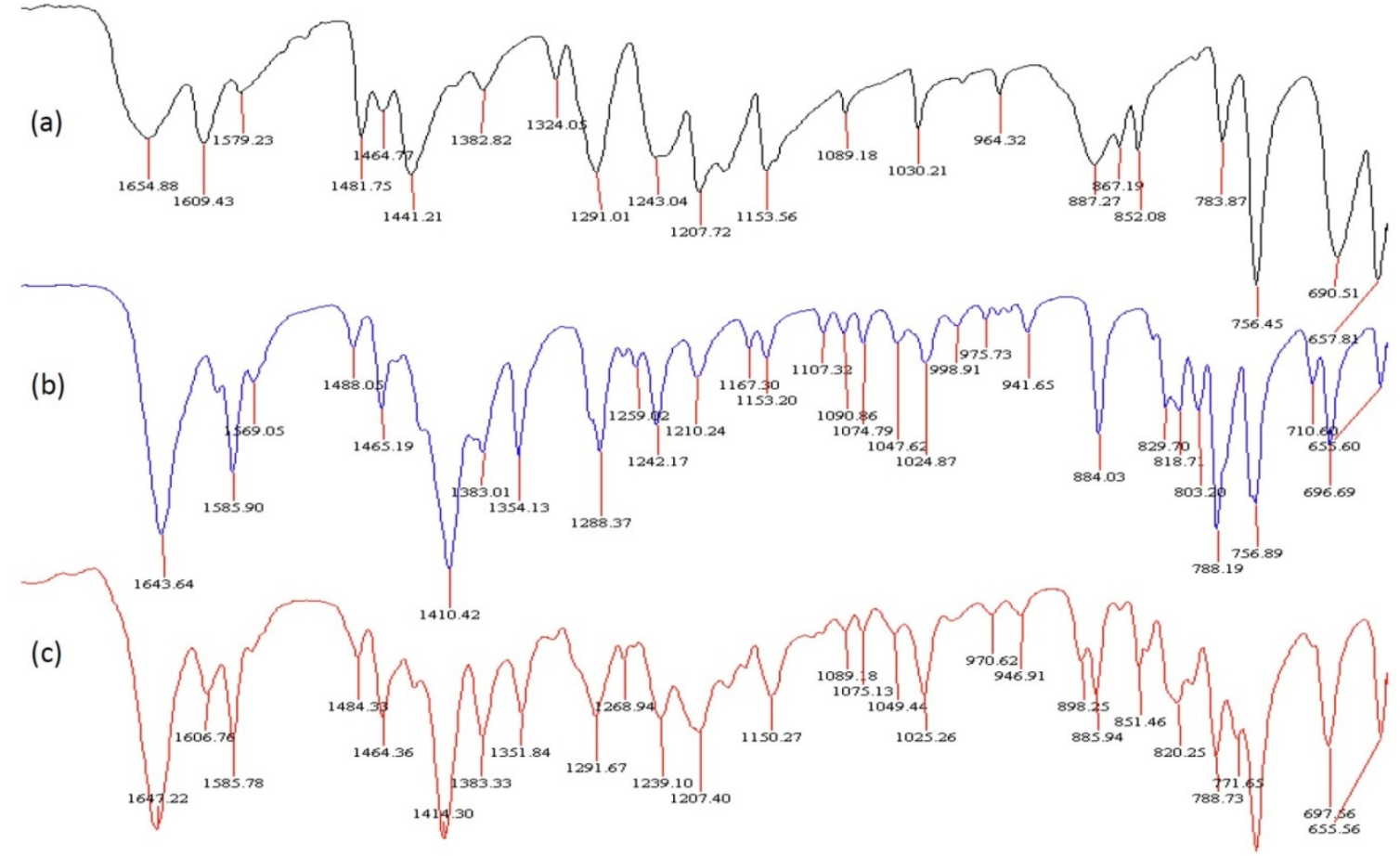

Figure 7: FTIR spectra of (a) SLI, (b) NV (c) NVSLI co-crystals. 
Citation: Samsodien H, Bapoo M, Doms TI, Harneker Z, Louw AS, et al. (2017) FTIR, Dissolution and Anti-viral Activity of Nevirapine Cocrystals. Pharm Anal Acta 8: 561. doi:10.4172/2153-2435.1000561

(a)

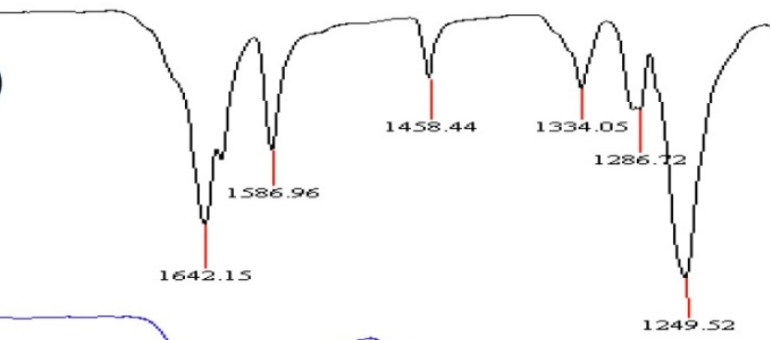

(b)

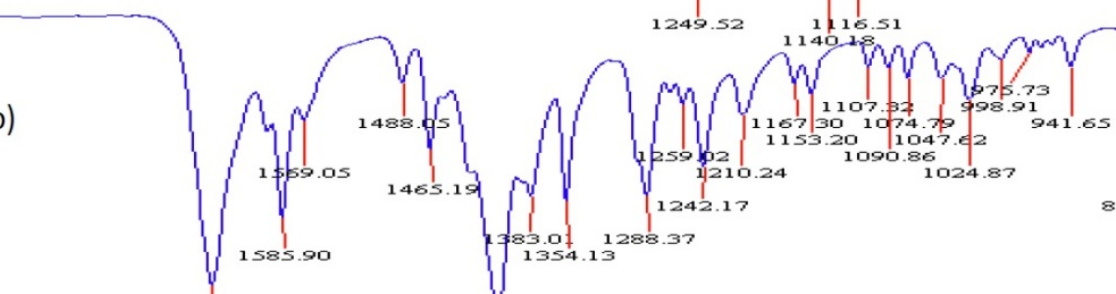

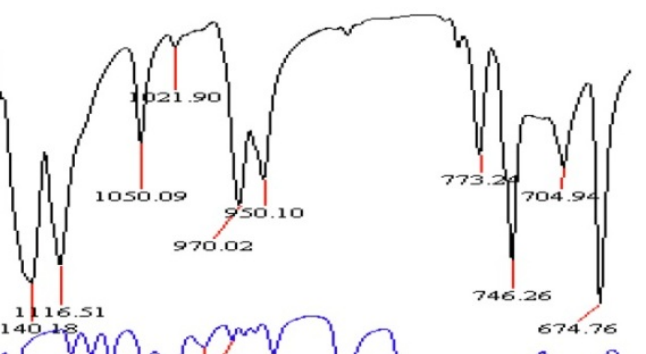

(c)

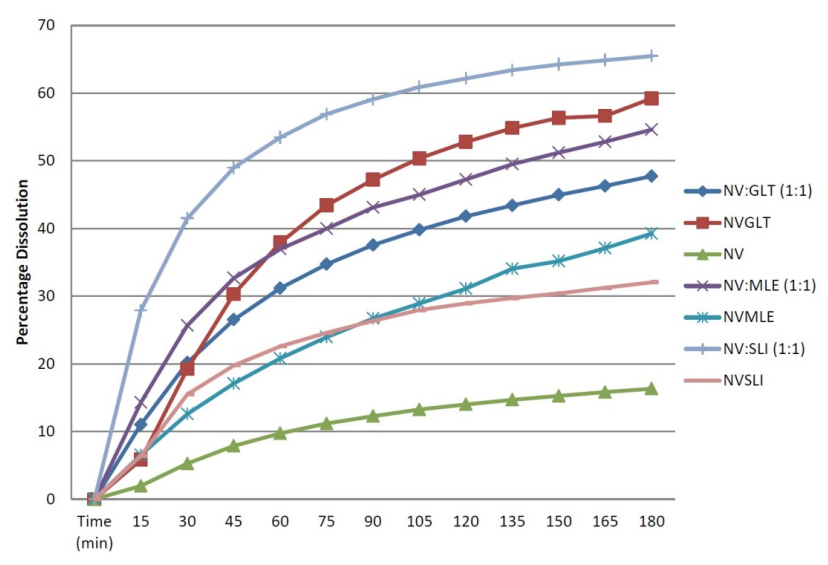

Figure 9: Dissolution profiles of selected NV co-crystals and mixtures.

A possible theory for the improved dissolution of the physical mixtures is based on the fact that the co-formers used in the dissolution study are weak acids and thus dissociate when added to water. The dissociation constant (pKa) is the degree to which an acid dissociates into water, the larger the pKa the smaller the extent of dissociation. Once an acid dissociates it lowers the $\mathrm{pH}$ of the solvent. $\mathrm{NV}$ is a weak base $(\mathrm{pKa}=2.8)$ which has better solubility in an acidic medium [9]. A lowering of the $\mathrm{pH}$ of the solvent by the dissociation of the co-formers in the mixes, will therefore increase the solubility of NV.
In both Figure 9 and Table 2, NV: SLI has the greatest total dissolution after 180 minutes. SLI has the smallest $\mathrm{pKa}$ value and thus it dissociates to a greater extent than the other co-formers. The greater extent of dissociation lowers the $\mathrm{pH}$ of the dissolution medium, facilitating an increase in solubility of NV.

\begin{tabular}{|l|l|l|l|}
\hline Co-former & pK, & $\begin{array}{l}\text { Mixture total \% } \\
\text { dissolution }\end{array}$ & $\begin{array}{l}\text { Co-crystal total } \\
\% \text { dissolution }\end{array}$ \\
\hline SLI & 3 & 65 & 32 \\
\hline GLT & 4.3 and 5.4 & 47 & 59 \\
\hline MLE & 1.9 and 6.3 & 54 & 39 \\
\hline
\end{tabular}

Table 2: $\mathrm{pK}_{\mathrm{a}}$ values and total percentage dissolution of NV physical mixtures and co-crystals.

MLE, being a diprotic acid, has two hydrogen atoms per molecule capable of dissociating. The dissociation of both hydrogen atoms does not occur at the same time. The pKa of 6.3 refers to the dissociation of only one hydrogen atom per MLE molecule. If both hydrogen atoms were to dissociate to the full extent, the pKa value would be 1.9. On the assumption that the first hydrogen atom fully dissociates and the second hydrogen only partially dissociates, the pKa value would fall in between the reference values. This explains why the total dissolution of NV: MLE is less than NV: SLI but more than NV: GLT.

GLT is also a diprotic acid with pKa values of 4.3 and 5.4. The higher pKa values of GLT compared to SLI means that it dissociates to a lesser extent and does not decrease the $\mathrm{pH}$ of the medium as significantly as SLI. This is proven by the total dissolution of NV: GLT being only $47 \%$ compared to $65 \%$ of NV: SLI. 
From Table 2 we can deduce that the lower the pKa of the co-former the better the solubility of $\mathrm{NV}$ when in a physical mixture. In comparison, a high $\mathrm{pKa}$ value of the co-former results in better solubility of NV when in the co-crystal form. This can be rationalized by a difference in the mechanism which causes the mixes and cocrystals to go into solution. Further research needs to be performed to understand these exact mechanisms. A possible explanation is that upon dissociation of the co-former and NV in co-crystal form, the molecules will both go into solution immediately. This is different from the mechanism which is displayed when the physical mixtures go into solution. Here the acidic co-formers go into solution first and lowers the $\mathrm{pH}$ of the solvent, creating a better environment for the $\mathrm{NV}$ to go into solution.

A possible theory for the improved dissolution of the co-crystals is based on a relationship between the melting point of the co-former and that of its co-crystal. The melting point of a co-crystal for a given API directly correlates with the melting points of the respective coformers (Figure 10) [1].

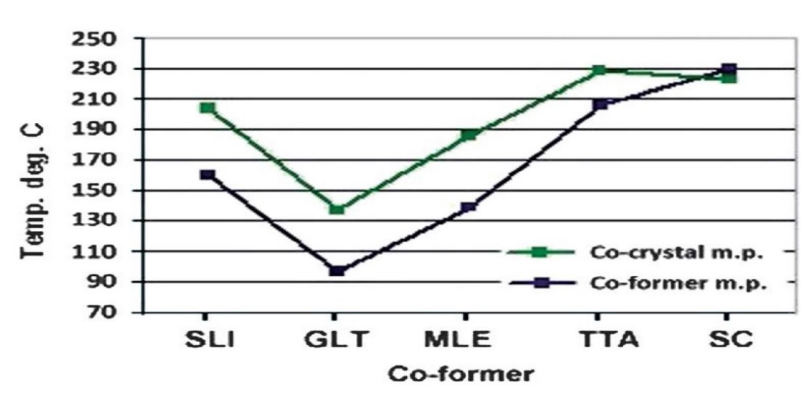

Figure 10: Melting points of NV co-crystals and the respective coformers [1].

Low thermal stability implies high water solubility [10]. NVGLT has the lowest melting point and the best total dissolution (Table 2). NVMLE has a significantly higher melting point than NVGLT, and hence a significantly lower total percent dissolution. NVSLI's melting point is only slightly higher than that of NVMLE, and its dissolution is also comparatively so. As can be seen from the results, the dissolution profile of each co-crystal correlates to its melting point.

\section{Antiviral testing}

In vitro antiviral testing of the co-crystals are necessary to confirm that the new crystalline form of $\mathrm{NV}$ has comparable or improved activity against $\mathrm{HIV}^{-1}$. The co-crystals showed no significant cytotoxicity to the $293 \mathrm{~T}$ cells, as percentage viability remained above $50 \%$ for all co-crystals tested (Figure 11). The cytotoxicity-50 (CC50) value is greater than the maximum concentration tested, indicating no cytotoxic effect to the $293 \mathrm{~T}$ cells. These concentration ranges were therefore used when screening for anti-HIV ${ }^{-1}$ activity.

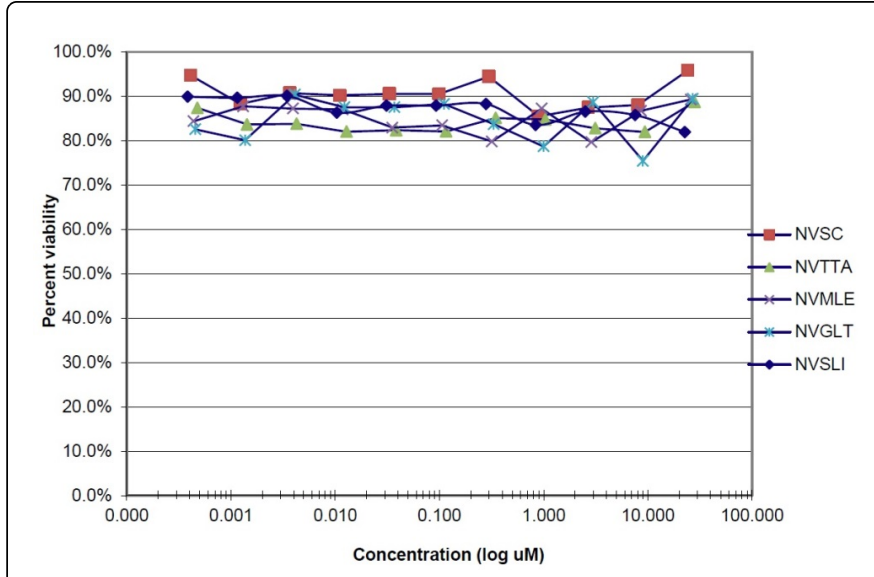

Figure 11: Cytotoxic effects of the NV co-crystals on the viability of $293 \mathrm{~T}$ cells in vitro.

When testing the antiviral activity, the NICD confirmed that neither the co-formers nor DMSO solvent inhibited $\mathrm{HIV}^{-1}$. This indicates that the inhibitory activity displayed by the co-crystals is directly as a result of the NV portion of the molecule. NVSC and NVSLI had an average $\mathrm{IC}_{50}$ value of $0.037 \mathrm{mM}$, which differed significantly from pure NV $(0.083 \mathrm{mM})$, with p-values of 0.002 . NVMLE and NVGLT had average $\mathrm{IC}_{50}$ values of $0.055 \mathrm{mM}$ and $0.054 \mathrm{mM}$ significantly different from pure NV with p-values of 0.026 and 0.019 , respectively. NVTTA had an average $\mathrm{IC}_{50}$ value of 0.072 , which does not differ significantly from pure NV, with a p-value of 0.416 (Figure 12).

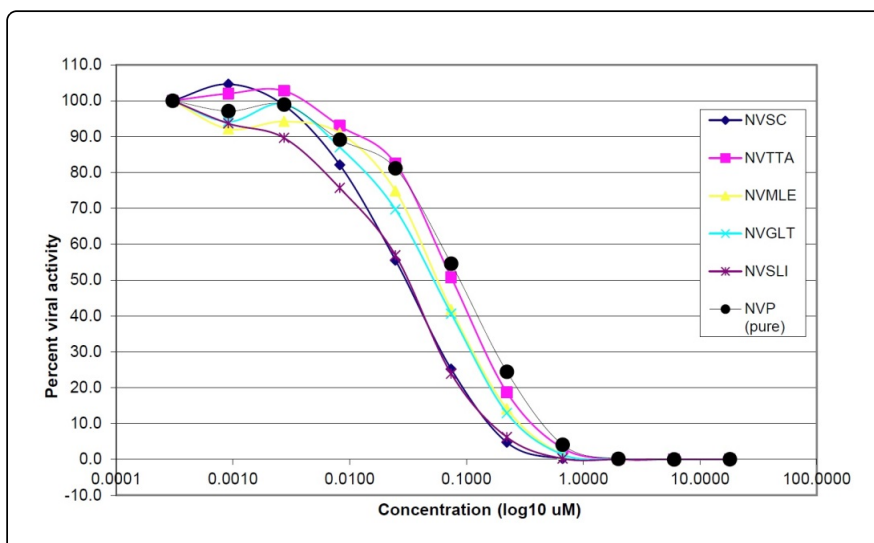

Figure 12: Antiviral activity of the NV co-crystals against HIV-1, compared to pure NV.

\section{Conclusion}

The results presented here indicate that FTIR is an appropriate analytical method of identifying co-crystals except for co-crystals with a 2:1 molecular ratio. Since the co-former and NV are held together by weak hydrogen bonds, peaks were expected to occur at the $\mathrm{C}=\mathrm{O}, \mathrm{O}-\mathrm{H}$ and $\mathrm{N}-\mathrm{H}$ bonds. The Spectrum software used in this study was only able to detect the $\mathrm{C}=\mathrm{O}$ bonds. The dissolution of $\mathrm{NV}$ was enhanced in the presence of individual co-formers, both in co-crystal form and as a physical mixture. NVGLT was the only co-crystal that yielded better results than its physical mixture. The solubility studies indicate that the 
Citation: Samsodien H, Bapoo M, Doms TI, Harneker Z, Louw AS, et al. (2017) FTIR, Dissolution and Anti-viral Activity of Nevirapine Cocrystals. Pharm Anal Acta 8: 561. doi:10.4172/2153-2435.1000561

Page 10 of 10

choice of co-formers for future research could possibly be based on pKa and melting point values. HPLC proved to be a more accurate and precise analytical method than UV spectrometry, producing more reliable results. Finally, the NVSC, NVSLI, NVMLE and NVGLT HIV ${ }^{-1}$ anti-viral activity differed significantly from pure $\mathrm{NV}$ compared to NVTTA.

\section{Acknowledgements}

The authors wish to thank the National Research Foundation for research support, the University of Cape Town Supramolecular group for the donation of the original co-crystals and the National Institute of Communicable Diseases for the antiviral study data. Any opinion, findings and conclusions or recommendations expressed in this material are those of the authors and therefore the NRF, UCT, NICD does not accept any liability in that regard.

\section{References}

1. Caira MR, Bourne SA, Samsodien H, Engel E, Liebenberg W, et al. (2011) Co-crystals of the antiretroviral nevirapine: crystal structures, thermal analysis and dissolution behaviour. Cryst Eng Comm 7: 2335-2596.

2. Guay LA, Musoke P, Fleming T, Bagenda D, Allen M, et al. (1999) ntrapartum and neonatal single-dose nevirapine compared with zidovudine for prevention of mother-to-child transmission of HIV-1 in Kampala, Uganda: HIVNET 012 randomised trial. The Lancet 354: 759-802.

3. Chadha R, Arora P, Saini A, Singh Jain D (2010) Solvated Crystalline Forms of Nevirapine: Thermoanalytical and Spectroscopic Studies. AAPS Pharm Sci Tech 11: 1328-1339.

4. Levin M (2001) Pharmaceutical Process Scale-Up, Marcel Dekker, Inc. New York p: 7.

5. Vishweshwar P, McMahon JA, Bis JA, Zaworotko MJ (2006) Pharmaceutical Co-Crystals. J Pharmac Sci 95: 499-516.

6. Shewale S, Shete AS, Doijad RC, Kadam SS, Patil VA, et al. (2015) Formulation and solid state characterization of nicotinamide-based cocrystals of fenofibrate. Indian J Pharm Sci 77: 328-334.

7. Basavoju S, Bostroin D, Velaga SP (2008) Indomethacin-Saccharin Cocrystal: Design, Synthesis and Preliminary Pharmaceutical Characterization. Pharma Res 25: 530-541.

8. British Pharmacopoeia (2005) British Pharmacopoeia Commission Secretariat. London, UK, 2005.

9. Cheeseman SH, Hattox SE, McLaughlin MM, Koup RA, Andrews C (1993) Pharmacokinetics of nevirapine: initial single-rising-dose study in humans. Antimicro Agen Chemother 37: 178-182.

10. McNamara DP, Childs SL, Giordano J, Iarriccio A, Cassidy A, et al. (2006) Use of a Glutaric Acid Cocrystal to Improve Oral Bioavailability of a Low Solubility API. Pharml Res 23: 1888-1897. 